Discrete Comput Geom 27:73-84 (2002)

DOI: $10.1007 / \mathrm{s} 00454-001-0053-8$

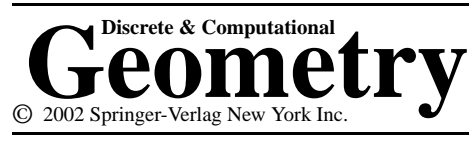

\title{
A Commutative Algebra for Oriented Matroids*
}

\author{
R. Cordovil \\ Departamento de Matemática, Instituto Superior Técnico, \\ Av. Rovisco Pais, 1049-001 Lisboa, Portugal \\ cordovil@math.ist.utl.pt
}

\begin{abstract}
Let $V$ be a vector space of dimension $d$ over a field $\mathbb{K}$ and let $\mathcal{A}$ be a central arrangement of hyperplanes in $V$. To answer a question posed by K. Aomoto, P. Orlik and $\mathrm{H}$. Terao construct a commutative $\mathbb{K}$-algebra $\mathbb{U}(\mathcal{A})$ in terms of the equations for the hyperplanes of $\mathcal{A}$. In the course of their work the following question naturally occurred:

- Is $\mathbb{U}(\mathcal{A})$ determined by the intersection lattice $L(\mathcal{A})$ of the hyperplanes of $\mathcal{A}$ ?

We give a negative answer to this question. The theory of oriented matroids gives rise to a combinatorial analogue of the algebra of Orlik-Terao, which is the main tool of our proofs.
\end{abstract}

\section{Introduction}

Let $\mathcal{M}=\mathcal{M}([n])($ resp. $\mathcal{M}=\mathcal{M}([n]))$ denote a matroid (resp. oriented matroid) of rank $r$ with ground set $[n]:=\{1,2, \ldots, n\}$. Let $V$ be a vector space of dimension $d$ over some field $\mathbb{K}$. A (central) arrangement (of hyperplanes) in $V, \mathcal{A}_{\mathbb{K}}=\left\{H_{1}, \ldots, H_{n}\right\}$, is a finite listed set of codimension one vector subspaces. Given an arrangement $\mathcal{A}_{\mathbb{K}}$ we suppose always chooses a family of linear forms $\left\{\theta_{H_{i}} \in V^{*}: H_{i} \in \mathcal{A}_{\mathbb{K}}, \operatorname{Ker}\left(\theta_{H_{i}}\right)=H_{i}\right\}$, where $V^{*}$ denotes the dual space of $V$. The product $Q(\mathcal{A})=\prod_{H \in \mathcal{A}} \theta_{H}$ is called the defining polynomial of $\mathcal{A}$. There is a matroid $\mathcal{M}\left(\mathcal{A}_{\mathbb{K}}\right)$ on the ground set $[n]$ determined by $\mathcal{A}_{\mathbb{K}}$ : a subset $D \subset[n]$ is a dependent set of $\mathcal{M}\left(\mathcal{A}_{\mathbb{K}}\right)$ iff there are scalars $\zeta_{i} \in \mathbb{K}, i \in D$, not all nulls, such that $\sum_{i \in D} \zeta_{i} \theta_{H_{i}}=0$. A circuit is a minimal dependent set with respect to inclusion. We denote by $L\left(\mathcal{A}_{\mathbb{K}}\right)$ the intersection lattice of $L\left(\mathcal{A}_{\mathbb{K}}\right)$ : i.e., the set of intersections of hyperplanes in $\mathcal{A}_{\mathbb{K}}$, partially ordered by reverse inclusion. Set $\mathfrak{M}\left(\mathcal{A}_{\mathbb{K}}\right)=V \backslash \bigcup_{H \in \mathcal{A}_{\mathbb{K}}} H$.

\footnotetext{
* This research was supported in part by FCT (Portugal) through program POCTI, the project SAPIENS/36563/99 and by DONET (European Union Contract ERBFMRX-CT98-0202).
} 
Aomoto suggested the study of the (graded) $\mathbb{K}$-vector space $\mathrm{AO}\left(\mathcal{A}_{\mathbb{K}}\right)$, generated by the basis $\left\{Q\left(\mathcal{B}_{\mathbb{I}}\right)^{-1}\right\}$, where $I$ is an independent set of $\mathcal{M}\left(\mathcal{A}_{\mathbb{K}}\right), \mathcal{B}_{\mathrm{I}}:=\left\{H_{i} \in \mathcal{A}_{\mathbb{K}}: i \in I\right\}$ and $Q\left(\mathcal{B}_{\mathrm{I}}\right)=\prod_{i \in I} \theta_{H_{i}}$ denotes the corresponding defining polynomial. In [1] it is conjectured that

$$
\operatorname{dim}\left(\mathrm{AO}\left(\mathcal{A}_{\mathbb{R}}\right)\right)=\text { number of chambers of } \mathfrak{M}\left(\mathcal{A}_{\mathbb{R}}\right) .
$$

To prove Aomoto's conjecture, Orlik and Terao have constructed in [8] a commutative $\mathbb{K}$-algebra, $\mathbb{U}\left(\mathcal{A}_{\mathbb{K}}\right)$, isomorphic to $\mathrm{AO}\left(\mathcal{A}_{\mathbb{K}}\right)$ as a graded $\mathbb{K}$-vector space in terms of the equations $\left\{\theta_{H}: H \in \mathcal{A}_{\mathbb{K}}\right\}$. The authors note that it is not clear whether $\mathbb{U}\left(\mathcal{A}_{\mathbb{K}}\right)$ itself depends only on the intersection lattice $L\left(\mathcal{A}_{\mathbb{K}}\right)$.

To every oriented matroid $\mathcal{M}$ we associate a commutative $\mathbb{Z}$-algebra, denoted by $\mathbb{A}(\mathcal{M})$. This algebra is the "combinatorial analogue" of the algebra of Orlik-Terao and it is the main tool to give a negative answer to the question of Orlik-Terao.

We use [9] and [10] as a general reference in matroid theory. We refer to [2] and [7] for good sources of the theory of oriented matroids and arrangements of hyperplanes, respectively.

\section{Two Commutative Algebras}

Let $\operatorname{IND}_{\ell}(\mathcal{M}) \subset\left(\begin{array}{c}{[n]} \\ \ell\end{array}\right)$ be the family of the independent sets of cardinal $\ell$ of the matroid $\mathcal{M}$ and set $\operatorname{IND}(\mathcal{M})=\bigcup_{\ell \in \mathbb{N}} \operatorname{IND}_{\ell}(\mathcal{M})$. We denote by $\mathfrak{C}=\mathfrak{C}(\mathcal{M})$ the set of circuits of $\mathcal{M}$. When the smallest element $\alpha$ of a circuit $C,|C|>1$, is deleted, the remaining set, $C \backslash \alpha$, is said to be a broken circuit. (Note that our definition is slightly different to the standard one. In the standard definition $C \backslash \alpha$ can be empty.) To shorten the notation the singleton set $\{x\}$ is denoted by $x$. A no broken circuit set of a matroid $\mathcal{M}$ is an independent subset of $[n]$ which does not contain any broken circuit. Let $\operatorname{NBC}_{\ell}(\mathcal{M}) \subset$ $\left(\begin{array}{c}{[n]} \\ \ell\end{array}\right)$ be the set of the no broken circuit sets of cardinal $\ell$ of $\mathcal{M}$. Set $\operatorname{NBC}(\mathcal{M})=$ $\bigcup_{\ell \in \mathbb{N}} \mathrm{NBC}_{\ell}(\mathcal{M})$. We denote by $L(\mathcal{M})$ the lattice of flats of $\mathcal{M}$. (We remark that the lattice map $\varphi: L\left(\mathcal{A}_{\mathbb{K}}\right) \rightarrow L\left(\mathcal{M}\left(\mathcal{A}_{\mathbb{K}}\right)\right)$, determined by the one-to-one correspondence $\varphi^{\prime}: H_{i} \longleftrightarrow\{i\}, i=1, \ldots, n$, is a lattice isomorphism.) Consider now an independent set $X$. Let $\operatorname{cl}_{\mathcal{M}}(X)$ be (or shortly $\operatorname{cl}(X)$ ) the closure of $X$ in $\mathcal{M}$. Pick an element $x \in \operatorname{cl}(X) \backslash X$. Let $C(X, x)$ denote the unique circuit of $\mathcal{M}$ contained in $X \cup x$. For every $X \in \operatorname{IND}(\mathcal{M})$, set

$$
\mathrm{EA}(X):=\{x \in \operatorname{cl}(X) \backslash X: x \text { is the minimum of } C(X, x) \text { and } C(X, x) \neq\{x\}\} .
$$

(The elements of $\operatorname{EA}(X)$ are usually called the externally active elements of $X$.) So, for every independent set $X$ of $\mathcal{M}, X \in \operatorname{NBC}(\mathcal{M})$ iff $\operatorname{EA}(X)=\emptyset$. If $\operatorname{EA}(X) \neq \emptyset$, let $\alpha(X)$ denote the smallest element of $\operatorname{EA}(X)$.

Here, every map $X:[n] \rightarrow\{+1,-1,0\} \subset \mathbb{Z}$ is called a signed set on $[n]$. Set $\boldsymbol{X}^{+}=\{\ell \in[n]: \boldsymbol{X}(\ell)=+1\}, \boldsymbol{X}^{-}=\{\ell \in[n]: \boldsymbol{X}(\ell)=-1\}$. We say that $\underline{X}:=\boldsymbol{X}^{+} \cup \boldsymbol{X}^{-}$ is the support of $\boldsymbol{X}$, or $\boldsymbol{X}$ is a signed set supporting $\underline{\boldsymbol{X}}$. A signed set $\boldsymbol{X}$ conforms to a signed set $\boldsymbol{Y}$ if $\boldsymbol{X}^{+} \subset \boldsymbol{Y}^{+}$and $\boldsymbol{X}^{-} \subset \boldsymbol{Y}^{-}$. For every $x \in[n]$ and signed set $\boldsymbol{X}$ let $\boldsymbol{X} \backslash x$ be the signed set on $[n] \backslash x$, conforming to $\boldsymbol{X}$ and supporting $\underline{\boldsymbol{X}} \backslash x$. We say that a signed set $\boldsymbol{X}$ is the union of the signed sets $\boldsymbol{X}_{1}, \ldots, \boldsymbol{X}_{m}$ if $\underline{\boldsymbol{X}}=\underline{\boldsymbol{X}}_{1} \cup \cdots \cup \underline{\boldsymbol{X}}_{m}$ and every $\boldsymbol{X}_{i}$ conforms to $X$. The reorientation on the subset $S \subset[n]$ of the signed set $X$ is the signed 
set, denoted ${ }_{-S} \boldsymbol{X}$, determined by the equalities

$$
\left({ }_{-S} \boldsymbol{X}\right)^{+}:=\left\{\boldsymbol{X}^{+} \backslash S\right\} \cup\left\{S \cap \boldsymbol{X}^{-}\right\} \quad \text { and } \quad\left({ }_{-S} \boldsymbol{X}\right)^{-}:=\left\{\boldsymbol{X}^{-} \backslash S\right\} \cup\left\{S \cap \boldsymbol{X}^{+}\right\} .
$$

The opposite of a signed set $\boldsymbol{X}$, denoted $-\boldsymbol{X}$, is the signed set $-\boldsymbol{X}={ }_{-[n]} \boldsymbol{X}$. An oriented matroid, denoted $\mathcal{M}$, is a matroid on the ground set $[n]$, denoted $\underline{\mathcal{M}}$, with an additional structure:

- To every circuit $C \in \mathfrak{C}(\underline{\mathcal{M}})$ is attached two opposite signed sets (signed circuits) $\boldsymbol{C}$ and $-\boldsymbol{C}$ supporting $\boldsymbol{C}$.

- The set of signed circuits of $\mathcal{M}$, denoted $\mathfrak{C}=\mathfrak{C}(\mathcal{M})$, verifies a convenient set of axioms, see page 103 of [2].

The set of all the union of signed circuits of $\mathcal{M}$ is called the set of the vectors of the oriented matroid. If $\mathbb{K}$ is an ordered field the arrangement $\mathcal{A}_{\mathbb{K}}$ determines an oriented matroid $\mathcal{M}\left(\mathcal{A}_{\mathbb{K}}\right)$ on the ground set $[n]$. Indeed let $C=\left\{i_{1}, \ldots, i_{m}\right\}, i_{1}<\cdots<i_{m}$, be a circuit of $\mathcal{M}\left(\mathcal{A}_{\mathbb{R}}\right)$. From the definitions we know that there are well determined scalars $\zeta_{i_{j}} \in \mathbb{K}^{*}, \zeta_{i_{1}}=1$, such that $\sum_{j=1}^{m} \zeta_{i_{j}} \theta_{H_{i_{j}}}=0$. Set $\boldsymbol{C}:[n] \rightarrow\{0,1,-1\} \subset \mathbb{Z}$ the signed set

$$
C(\ell)= \begin{cases}+1 & \text { if } \quad \ell \in C \quad \text { and } \quad \lambda_{\ell}>0 \\ -1 & \text { if } \quad \ell \in C \quad \text { and } \quad \lambda_{\ell}<0 \\ 0 & \text { if } \ell \notin C .\end{cases}
$$

By definition $C$ is one of the two opposite signed circuits of $\mathcal{M}\left(\mathcal{A}_{\mathbb{K}}\right)$ supporting $C$. Note that $\mathcal{M}\left(\mathcal{A}_{\mathbb{K}}\right)=\underline{\mathcal{M}}\left(\mathcal{A}_{\mathbb{K}}\right)$. The reorientation on the subset $S \subset[n]$, of the oriented matroid $\mathcal{M}$, is the oriented matroid, denoted ${ }_{-S} \mathcal{M}$, such that $\mathfrak{C}\left({ }_{-S} \mathcal{M}\right):=\left\{{ }_{S} \boldsymbol{C}: \boldsymbol{C} \in\right.$ $\mathfrak{C}(\mathcal{M})\}$. We say also that $\mathfrak{C}\left({ }_{-} \mathcal{M}\right)$ is the reorientation on the subset $S$ of $\mathfrak{C}(\mathcal{M})$. (The concept of "reorientation" is the combinatorial analogue of the notion of "nonsingular projective permissible transformation".)

Fix a set $E:=\left\{e_{1}, \ldots, e_{n}\right\}$ and let $\mathrm{K}$ be a commutative ring with unity 1 . Let $\mathrm{K}[E]$ denote the commutative free K-algebra given by the generators $E \cup\{1\}$. For every $X \subset[n]$, set $e_{X}:=\prod_{i \in X} e_{i}, e_{\emptyset}:=1$.

Definition 2.1 [8, Definition 2.2 and Proposition 2.3]. Consider the map

$$
\partial: \mathfrak{C}\left(\mathcal{M}\left(\mathcal{A}_{\mathbb{K}}\right)\right) \rightarrow \mathbb{K}[E], \quad C \mapsto \sum_{j=1}^{m} \zeta_{i_{j}} e_{C \backslash i_{j}}
$$

where $C=\left\{i_{1}, \ldots, i_{m}\right\}, i_{1}<\cdots<i_{m}$, and $\sum_{j=1}^{m} \zeta_{i_{j}} \theta_{H_{i_{j}}}=0, \zeta_{i_{1}}=1 . \mathbb{U}\left(\mathcal{A}_{\mathbb{K}}\right)$ is the (commutative) $\mathbb{K}$-algebra given by the generators $1, e_{1}, \ldots, e_{n}$, and the relations:

$\circ e_{i} e_{j}=e_{j} e_{i}, \forall i, j=1, \ldots, n$,

$\circ e_{i}^{2}=0, \forall i=1, \ldots, n$,

○ $\partial(C)=0, \forall C \in \mathfrak{C}\left(\mathcal{M}\left(\mathcal{A}_{\mathbb{K}}\right)\right)$.

We call $\mathbb{U}\left(\mathcal{A}_{\mathbb{K}}\right)$ the Orlik-Terao algebra of $\mathcal{A}_{\mathbb{K}}$.

Now, we introduce the "combinatorial analogue" of the Orlik-Terao algebra. 
Definition 2.2. Consider the map

$$
\widetilde{\partial}: \mathfrak{C}(\mathcal{M}) \rightarrow \mathbb{Z}[E], \quad C \mapsto \sum_{j=1}^{m} C\left(i_{j}\right) e_{C \backslash i_{j}},
$$

where $C=\left\{i_{1}, \ldots, i_{m}\right\}, i_{1}<\cdots<i_{m}$, and $\boldsymbol{C} \in \mathfrak{C}(\mathcal{M})$ is the signed circuit supporting $C$, such that $\boldsymbol{C}\left(i_{1}\right)=1 . \mathbb{A}(\mathcal{M})$ is the (commutative) $\mathbb{Z}$-algebra given by the generators $1, e_{1}, \ldots, e_{n}$, and the relations:

$\circ e_{i} e_{j}=e_{j} e_{i}, \forall i, j=1, \ldots, n$,

$\circ e_{i}^{2}=0, \forall i=1, \ldots, n$,

$\circ e_{i}=0$, if $i$ is a loop of $\mathcal{M}$,

○ $\widetilde{\partial}(C)=0, \forall C \in \mathfrak{C}(\mathcal{M}),|C|>1$.

We call $\mathbb{A}(\mathcal{M})$ the algebra of the oriented matroid $\mathcal{M}$.

For every $X \subset[n]$, we denote by $[X]_{\mathbb{A}}$ (resp. $[X]_{\mathbb{U}}$ ), or shortly by $[X]$ or even $e_{X}$ when no confusion will result, the residue class in $\mathbb{A}(\mathcal{M})$ (resp. $\mathbb{U}\left(\mathcal{A}_{\mathbb{K}}\right)$ ) determined by the element $e_{X}$. Note that $\mathbb{A}(\mathcal{M}) \cong \mathbb{A}(\mathcal{M} \backslash x)$ if $x$ is a loop or $x$ is parallel to some other element of $\mathcal{M}$. So in what follows we suppose that $\mathcal{M}$ is a simple matroid. For every circuit $C \in \mathfrak{C}(\mathcal{M})$, we have $[C]_{\mathbb{A}}=0$. To see this, pick an element $x \in C$ if $|C|>1$. Then $0=e_{x} \cdot \widetilde{\partial}\left(e_{C}\right)= \pm e_{C}$. We conclude that if $[X]_{\mathbb{A}} \neq 0$, then $X$ is an independent set of $\mathcal{M}$.

The "abstract algebra" $\mathbb{A}(\mathcal{M})$ has a canonical grading.

Proposition 2.3. Set $\mathbb{A}_{\ell}=\mathbb{A}_{\ell}(\mathcal{M})$ be the submodule of $\mathbb{A}(\mathcal{M})$ generated by the elements $\left\{[X]_{\mathbb{A}}: X \in \operatorname{IND}_{\ell}(\mathcal{M})\right\}$. The grading $\mathbb{A}(\mathcal{M})=\bigoplus_{\ell \in \mathbb{N}} \mathbb{A}_{\ell}(\mathcal{M})$ is canonical, i.e., it is independent of the knowledge of the oriented matroid $\mathcal{M}$.

Proof. We know that $\mathbb{A}_{\ell}(\mathcal{M})=(0)$, for all $\ell>r$. If $\mathbb{A}(\mathcal{M})=\mathbb{A}_{0}=\mathbb{Z}$ (i.e., $\left.r=0\right)$ the result is clear. Suppose that $\mathbb{A}(\mathcal{M}) \neq \mathbb{Z}$. Note that $\mathbb{A}_{r}=\{x \in \mathbb{A}(\mathcal{M}): x \cdot y=0, \forall y \in$ $\mathbb{A}(\mathcal{M}) \backslash \mathbb{Z}\}$. If we know the modules $\mathbb{A}_{r}, \ldots, \mathbb{A}_{r-i}$ and $\mathbb{A}^{\langle i+1\rangle}:=\mathbb{A}_{r} \oplus \cdots \oplus \mathbb{A}_{r-i} \neq$ $\mathbb{A}(\mathcal{M})$, (i.e., $r-i>1$ ) the module $\mathbb{A}_{r-i-1}, i=0, \ldots, r-2$, can be defined recursively by

$$
\mathbb{A}_{r-i-1}=\left\{x \in \mathbb{A}(\mathcal{M}): x \cdot y \in \mathbb{A}^{\langle i+1\rangle}, \forall y \in \mathbb{A}(\mathcal{M}) \backslash \mathbb{Z}\right\} / \mathbb{A}^{\langle i+1\rangle}
$$

Proposition 2.4. For every $x \in[n]$ there is a unique epimorphism of $\mathbb{Z}$-modules $\mathfrak{p}_{x}: \mathbb{A}(\mathcal{M}) \rightarrow \mathbb{A}(\mathcal{M} / x)$, such that, for every $I \in \operatorname{IND}(\mathcal{M})$, we have

$$
\mathfrak{p}_{x}\left(e_{I}\right):= \begin{cases}e_{I \backslash x} & \text { if } x \in I \in \operatorname{IND}(\mathcal{M}), \\ 0 & \text { otherwise. }\end{cases}
$$

Proof. It is enough to prove that

$$
\mathfrak{p}_{x}\left(e_{X} \cdot \widetilde{\partial}(C)\right)=0, \quad \forall X \subset[n], \quad \forall C \in \mathfrak{C}(\mathcal{M}) .
$$


We can suppose that $X \cap C=\emptyset$ and $x \in X \cup C$. Let $\boldsymbol{Y}$ be a signed set on [ $n]$ supporting $\underline{\boldsymbol{Y}}=\left\{i_{1}, \ldots, i_{m}\right\}$. For convenience of notation set

$$
\tilde{\partial}_{\boldsymbol{Y}}(\underline{\boldsymbol{Y}}):=\sum_{j=1}^{m} \boldsymbol{Y}\left(i_{j}\right) e_{\underline{Y} \backslash i_{j}} \in \mathbb{Z}[E] .
$$

Let $C$ be one of the opposite signed circuits supporting $C$. Remember that the signed set $\boldsymbol{C} \backslash x$ on $[n] \backslash x$ is a vector (union of signed circuits) of $\mathcal{M} / x$. So we have

$$
\mathfrak{p}_{x}\left(e_{X} \cdot \tilde{\partial}(C)\right)=\left\{\begin{array}{lll} 
\pm e_{X} \cdot \widetilde{\partial}_{C \backslash x}(C \backslash x)=0 & \text { if } & x \in C, \\
\pm e_{X \backslash x} \cdot \widetilde{\partial}_{C}(C)=0 & \text { if } & x \in X .
\end{array}\right.
$$

Corollary 2.5. For every subset $X=\left\{i_{1}, \ldots, i_{m}\right\} \subset[n]$, the following two conditions are equivalents:

$\circ X$ is an independent set of $\mathcal{M}$,

$\circ[X]_{\mathbb{A}}=e_{i_{1}} e_{i_{2}} \cdots e_{i_{m}} \neq 0$.

Proof. It remains to prove that if $X$ is an independent set of $\mathcal{M}$, then $[X]_{\mathbb{A}} \neq 0$. We prove by induction on $n$. We know that $[\emptyset]_{\mathbb{A}}=1$. Suppose that the implication is true for all the matroids with at most $n-1$ elements. Let $X,|X|>0$, be an independent set of $\mathcal{M}$ and pick an element $x \in X$. Suppose for a contradiction that $[X]_{\mathbb{A}}=0 . X \backslash x$ is an independent set of $\mathcal{M} / x$. From Proposition 2.4 we conclude that $0=\mathfrak{p}_{x}\left([X]_{\mathbb{A}}\right)=$ $[X \backslash x]_{\mathbb{A}(\mathcal{M} / x)}$, a contradiction with the induction hypothesis.

Proposition 2.6. For every $x \in[n]$ there is a unique morphism of $\mathbb{Z}$-modules, $\mathfrak{i}_{x}: \mathbb{A}$ $(\mathcal{M} \backslash x) \rightarrow \mathbb{A}(\mathcal{M})$, such that, for every $I \in \operatorname{IND}(\mathcal{M} \backslash x)$, we have $\mathfrak{i}_{x}\left(e_{I}\right)=e_{I}$.

Proof. The map $\mathfrak{i}_{x}$ is well determined. Indeed from Corollary 2.5 we know that

$$
\mathfrak{i}_{x}\left(e_{I} \cdot \tilde{\partial}(C)\right)=e_{I} \cdot \tilde{\partial}(C)=0, \quad \forall I \in \operatorname{IND}(\mathcal{M} \backslash x), \quad \forall C \in \mathfrak{C}(\mathcal{M} \backslash x) .
$$

Set $\boldsymbol{n} \boldsymbol{b} \boldsymbol{c}_{\ell}:=\left\{[I]_{\mathbb{A}}: I \in \mathrm{NBC}_{\ell}(\mathcal{M})\right\}$ and $\boldsymbol{n} \boldsymbol{b c}:=\bigcup_{\ell=0} \boldsymbol{n} \boldsymbol{b} \boldsymbol{c}_{\ell}$. Set $\mathcal{M}^{\prime}=\mathcal{M} \backslash x$, $\mathcal{M}^{\prime \prime}=\mathcal{M} / x, \mathbb{A}:=\mathbb{A}(\mathcal{M}), \mathbb{A}^{\prime}:=\mathbb{A}\left(\mathcal{M}^{\prime}\right)$ and $\mathbb{A}^{\prime \prime}:=\mathbb{A}\left(\mathcal{M}^{\prime \prime}\right)$. Consider an independent set $X \in \operatorname{IND}_{\ell}(\mathcal{M})_{\tilde{\partial}}$, suppose that $X \notin \operatorname{NBC}_{\ell}(\mathcal{M})$, and set $\alpha=\alpha(X)$. Making use of the definition of $\widetilde{\partial}(C(X, \alpha))$ we can express the element $[X]_{\mathbb{A}}$ as a linear combination of the elements $\left\{\left[X_{x}\right]_{\mathbb{A}}: X_{x}=X \backslash x \cup \alpha, x \in C(X, \alpha) \backslash \alpha\right\}$. We claim that $\mathrm{EA}\left(X_{x}\right) \subset \mathrm{EA}(X) \backslash \alpha$. Indeed, suppose that $\beta \in \mathrm{EA}\left(X_{x}\right)$. If $\alpha \notin C\left(X_{x}, \beta\right)=C(X, \beta)$, then we have $\beta \in \operatorname{EA}(X) \backslash \alpha$. If $\alpha \in C\left(X_{x}, \beta\right)$ we have $\beta<\alpha$ and from the elimination axiom for circuits there is a circuit $C^{\prime}$ such that $\beta \in C^{\prime} \subset\left(C\left(X_{x}, \beta\right) \cup C(X, \alpha)\right) \backslash \alpha$. So $C^{\prime}=C(X, \beta), \beta$ is the smallest element $C(X, \beta)$ and the claim follows. By the iteration of this process we conclude that $[X]_{\mathbb{A}}$ can be expressed linearly in term of elements of $\boldsymbol{n} \boldsymbol{b} \boldsymbol{c}_{\ell}=\left\{[I]_{\mathbb{A}} \in \mathrm{IND}_{\ell}(\mathcal{M}): \operatorname{EA}(I)=\emptyset\right\}$. So $\boldsymbol{n} \boldsymbol{b} \boldsymbol{c}_{\ell}$ is a generating set of the $\mathbb{Z}$-module $\mathbb{A}_{\ell}(\mathcal{M})$. Now we are able to prove the main results of this section. 
Theorem 2.7. For every element $x$ of $\mathcal{M}$, there is a split short exact sequence of modules

$$
0 \rightarrow \mathbb{A}(\mathcal{M} \backslash x) \stackrel{\mathfrak{i}_{x}}{\longrightarrow} \mathbb{A}(\mathcal{M}) \stackrel{\mathfrak{p}_{x}}{\longrightarrow} \mathbb{A}(\mathcal{M} / x) \rightarrow 0 .
$$

We postpone the proof of the theorem. The following corollary is an important direct consequence of the above theorem. Corollary 2.8 is similar to a well-known result concerning the algebras of Orlik-Solomon, Theorem 3.55 of [7].

Corollary 2.8. Suppose that the sequence (2.3) is exact for all the matroids with at most $n$ elements. Then $\boldsymbol{n b c}(\mathcal{M})$ is a basis of the module $\mathbb{A}(\mathcal{M})$.

Proof. We prove by induction on $n$. If $n=0$ we know that $\mathbb{A}(\mathcal{M}(\emptyset))=\mathbb{Z}$ and $\boldsymbol{n b c}(\mathcal{M}(\emptyset))=\{1\}$. Suppose that $n>0$ and that the result is true for all the matroids with at most $n-1$ elements. By a reordering of the elements of the matroid $\mathcal{M}$ we can suppose that $x=n$. It is clear that

$$
\operatorname{NBC}\left(\mathcal{M}^{\prime}\right)=\{X: X \subset[n-1] \text { and } X \in \operatorname{NBC}(\mathcal{M})\} .
$$

From the induction hypothesis we know that $\boldsymbol{n b} \boldsymbol{c}\left(\mathcal{M}^{\prime}\right)=\left\{[X]_{\mathbb{A}^{\prime}}: X \in \operatorname{NBC}\left(\mathcal{M}^{\prime}\right)\right\}$ and $\boldsymbol{n b c}\left(\mathcal{M}^{\prime \prime}\right)=\left\{[X]_{\mathbb{A}^{\prime \prime}}: X \in \mathrm{NBC}\left(\mathcal{M}^{\prime \prime}\right)\right\}$ are bases of $\mathbb{A}^{\prime}$ and $\mathbb{A}^{\prime \prime}$, respectively. The minimal broken circuits of $\mathcal{M} / n$ are the minimal sets $X$ such that either $X$ or $X \cup\{n\}$ is a broken circuit of $\mathcal{M}$ (see Proposition 3.2.e of [4]). Then

$$
\begin{gathered}
\operatorname{NBC}\left(\mathcal{M}^{\prime \prime}\right)=\{X: X \subset[n-1] \text { and } X \cup\{n\} \in \operatorname{NBC}(\mathcal{M})\} \quad \text { and } \\
\operatorname{NBC}(\mathcal{M})=\operatorname{NBC}\left(\mathcal{M}^{\prime}\right) \uplus\left\{I \cup n: I \in \operatorname{NBC}\left(\mathcal{M}^{\prime \prime}\right)\right\} .
\end{gathered}
$$

We know that $\boldsymbol{n} \boldsymbol{b} \boldsymbol{c}(\mathcal{M})=\left\{[X]_{\mathbb{A}}: X \in \operatorname{NBC}(\mathcal{M})\right\}$ is a generating set of $\mathbb{A}$. So Corollary 2.8 follows from the exactness of sequence (2.3).

Theorem 2.7 is a consequence of Lemmas 2.10-2.12 below.

Lemma 2.9. Suppose that sequence (2.3) is exact for all the matroids with at most $n-1$ elements. Then for every $x \in[n]$, there is an exact sequence of $\mathbb{Z}$-modules $\mathbb{A}^{\prime} \stackrel{\mathfrak{i}_{x}}{\longrightarrow} \mathbb{A} \stackrel{\mathfrak{p}_{x}}{\longrightarrow} \mathbb{A}^{\prime \prime} \rightarrow 0$.

Proof. From the definitions we know that $\mathfrak{p}_{x} \circ \mathfrak{i}_{x}$, is the null map so $\operatorname{Im}\left(\mathfrak{i}_{x}\right) \subset \operatorname{Ker}\left(\mathfrak{p}_{x}\right)$. It remains to prove the inclusion $\operatorname{Ker}\left(\mathfrak{p}_{x}\right) \subset \operatorname{Im}\left(\mathfrak{i}_{x}\right)$. By a reordering of the elements of $[n]$ we can suppose that $x=n$. Suppose that $\sum_{i=1}^{m} \zeta_{i}\left[I_{i}\right]_{\mathbb{A}} \in \operatorname{Ker}\left(\mathfrak{p}_{n}\right),\left[I_{i}\right]_{\mathbb{A}} \in \boldsymbol{n b c}(\mathcal{M}), \zeta_{i} \in$ $\mathbb{Z}$, and set

$$
\sum_{i=1}^{m} \zeta_{i}\left[I_{i}\right]_{\mathbb{A}}=\sum_{I_{i^{\prime}} \supsetneqq n} \zeta_{i^{\prime}}\left[I_{i^{\prime}}\right]_{\mathbb{A}}+\sum_{I_{i^{\prime \prime}} \ni n} \zeta_{i^{\prime \prime}}\left[I_{i^{\prime \prime}}\right]_{\mathbb{A}}, \quad i^{\prime}, i^{\prime \prime} \in\{1, \ldots, m\} .
$$

So,

$$
\mathfrak{p}_{n}\left(\sum_{i=1}^{m} \zeta_{i}\left[I_{i}\right]_{\mathbb{A}}\right)=\sum_{I_{i^{\prime \prime}} \ni n} \zeta_{i^{\prime \prime}}\left[I_{i^{\prime \prime}} \backslash n\right]_{\mathbb{A}^{\prime \prime}}=0
$$


From (2.4) we conclude that $\left[I_{i^{\prime \prime}} \backslash n\right]_{\mathbb{A}^{\prime \prime}} \in \boldsymbol{n b \boldsymbol { c }}\left(\mathbb{A}^{\prime \prime}\right)$. From Corollary 2.8 we know that $\boldsymbol{n b c}\left(\mathbb{A}^{\prime \prime}\right)$ is a basis of $\mathbb{A}^{\prime \prime}$. So, (2.6) implies that $\zeta_{i^{\prime \prime}}=0$ for every $i^{\prime \prime}$. Hence $\sum_{i=1}^{m} \zeta_{i}\left[I_{i}\right]_{\mathbb{A}}=$ $\sum_{I_{i^{\prime}} \supsetneqq n} \zeta_{i^{\prime}}\left[I_{i^{\prime}}\right]_{\mathbb{A}}=i_{n}\left(\sum \zeta_{i^{\prime}}\left[I_{i^{\prime}}\right]_{\mathbb{A}^{\prime}}\right) \in \operatorname{Im}\left(\mathfrak{i}_{n}\right)$.

Lemma 2.10. Suppose that sequence (2.3) is exact for all the matroids with at most $n-1$ elements. Let $\mathbf{a}$ be an element of the module $\mathbb{A}(\mathcal{M})=\mathbb{Z} \oplus \mathbb{A}_{1} \oplus \cdots \oplus \mathbb{A}_{r}$. Then $\mathbf{a} \in \mathbb{Z}$ iff $\mathfrak{p}_{x}(\mathbf{a})=0$, for every $x \in[n]$.

Proof. From (2.1) we see that if $\mathbf{a}=\zeta[\emptyset]_{\mathbb{A}} \in \mathbb{A}_{0}(=\mathbb{Z})$, then $\mathfrak{p}_{x}(\mathbf{a})=0$, for all $x \in[n]$. We prove that $\left(\mathfrak{p}_{x}(\mathbf{a})=0, \forall x \in[n]\right) \Longrightarrow \mathbf{a} \in \mathbb{Z}$. Suppose that $\mathfrak{p}_{x}(\mathbf{a})=0, \forall x \in[n]$. From Lemma 2.9 we know that

$$
\mathbf{a} \in \bigcap_{x \in[n]} \operatorname{Ker}\left(\mathfrak{p}_{x}\right)=\bigcap_{x \in[n]} \operatorname{Im}\left(\mathfrak{i}_{x}\right)=\mathbb{Z} .
$$

Lemma 2.11. Suppose that sequence (2.3) is exact for all the matroids with at most $n-1$ elements. Then the sequence $0 \rightarrow \mathbb{A}^{\prime} \stackrel{\mathfrak{i}_{x}}{\longrightarrow} \mathbb{A}$ is exact.

Proof. The case $n=1$ is trivial. Suppose that $n>1$. It is well known that $\mathcal{M} \backslash x / y=$ $\mathcal{M} / y \backslash x$, for every pair of elements $x, y \in[n], x \neq y$. Consider the epimorphism

$$
\mathfrak{p}_{y}^{\prime}: \mathbb{A}(\mathcal{M} \backslash x) \rightarrow \mathbb{A}(\mathcal{M} \backslash x / y) .
$$

Consider also the monomorphism $\mathfrak{i}_{x}^{\prime}: \mathbb{A}(\mathcal{M} \backslash x / y) \rightarrow \mathbb{A}(\mathcal{M} / y)$. It is easy to check that the following diagram of modules is commutative:

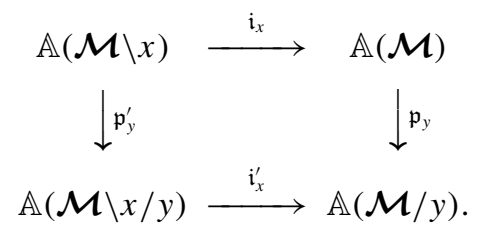

We prove the implication $\mathfrak{i}_{x}(\mathbf{a})=\mathfrak{i}_{x}(\mathbf{b}) \Longrightarrow \mathbf{a}=\mathbf{b}$, for every pair $\mathbf{a}, \mathbf{b} \in \mathbb{A}(\mathcal{M} \backslash x)$. We know that

$$
\begin{gathered}
\mathfrak{i}_{x}(\mathbf{a})=\mathfrak{i}_{x}(\mathbf{b}) \quad \Longrightarrow \quad\left(\mathfrak{p}_{y} \circ \mathfrak{i}_{x}(\mathbf{a})=\mathfrak{p}_{y} \circ \mathfrak{i}_{x}(\mathbf{b}), \forall y \in[n] \backslash x\right), \\
\mathfrak{p}_{y} \circ \mathfrak{i}_{x}(\mathbf{a})=\mathfrak{i}_{x}^{\prime} \circ \mathfrak{p}_{y}^{\prime}(\mathbf{a}) \quad \text { and } \quad \mathfrak{p}_{y} \circ \mathfrak{i}_{x}(\mathbf{b})=\mathfrak{i}_{x}^{\prime} \circ \mathfrak{p}_{y}^{\prime}(\mathbf{b}), \quad \forall y \in[n] \backslash x, \\
\mathfrak{i}_{x}^{\prime} \circ \mathfrak{p}_{y}^{\prime}(\mathbf{a})=\mathfrak{i}_{x}^{\prime} \circ \mathfrak{p}_{y}^{\prime}(\mathbf{b}) \quad \Longleftrightarrow \quad \mathfrak{p}_{y}^{\prime}(\mathbf{a})=\mathfrak{p}_{y}^{\prime}(\mathbf{b}), \quad \forall y \in[n] \backslash x .
\end{gathered}
$$

From Lemma 2.10 we know that $\left(\mathfrak{p}_{y}^{\prime}(\mathbf{a})=\mathfrak{p}_{y}^{\prime}(\mathbf{b}), \forall y \in[n] \backslash x\right) \Leftrightarrow \mathbf{a}-\mathbf{b}=\zeta \in \mathbb{Z}$. Then we have $0=\mathfrak{i}_{x}(\mathbf{a})-\mathfrak{i}_{x}(\mathbf{b})=\mathfrak{i}_{x}(\zeta)=\zeta$ and so $\mathbf{a}=\mathbf{b}$.

Lemma 2.12. Suppose that sequence (2.3) is exact for all the matroids with at most $n$ elements. Then sequence (2.3) for the matroid $\mathcal{M}([n])$ splits, i.e., there is a morphism of modules $\mathfrak{p}_{x}^{-1}: \mathbb{A}^{\prime \prime} \rightarrow \mathbb{A}$ such that $\mathfrak{p}_{x} \circ \mathfrak{p}_{x}^{-1}$ is the identity map and

$$
\mathbb{A}(\mathcal{M})=\mathfrak{i}_{x}(\mathbb{A}(\mathcal{M} \backslash x)) \oplus \mathfrak{p}_{x}^{-1}(\mathbb{A}(\mathcal{M} / x)) .
$$


Proof. We can suppose that $x=n$. From Corollary 2.8, we know that $\boldsymbol{n b \boldsymbol { c }}\left(\mathcal{M}^{\prime}\right)$ and $\boldsymbol{n b c}\left(\mathcal{M}^{\prime \prime}\right)$ are bases of $\mathbb{A}^{\prime}$ and $\mathbb{A}^{\prime \prime}$, respectively. There is a morphism of modules $\mathfrak{p}_{n}^{-1}: \mathbb{A}^{\prime \prime} \rightarrow \mathbb{A}$ well determined by the conditions $\mathfrak{p}_{n}^{-1}\left([I]_{\mathbb{A}^{\prime \prime}}\right):=[I \cup n]_{\mathbb{A}}$ for all $I \in$ $\operatorname{NBC}\left(\mathcal{M}^{\prime \prime}\right)$. It is clear that $\mathfrak{p}_{n} \circ \mathfrak{p}_{n}^{-1}$ is the identity map. From (2.5) we conclude that the exact sequence (2.3) splits.

Remark 2.13. With some adjustments, our techniques also give proofs of Proposition 2.3, Corollary 2.5, Theorem 2.7 and Corollary 2.8 for the algebra of Orlik-Terao.

\section{Applications}

Proposition 3.1. Let $V$ be a vector space of dimension d over an ordered field $\mathbb{K}$, and let $\mathcal{A}$ be a central arrangement of hyperplanes in $V$. Consider the algebras $\mathbb{U}=\mathbb{U}\left(\mathcal{A}_{\mathbb{K}}\right)$ and $\mathbb{A}=\mathbb{A}\left(\mathcal{M}\left(\mathcal{A}_{\mathbb{K}}\right)\right)$ and let $\boldsymbol{n b c}(\mathbb{U})$ and $\boldsymbol{n b c}(\mathcal{M})$ be the corresponding no broken circuit bases. For a given $X \subset[n]$, suppose that

(1) $[X]_{\mathbb{U}}=\sum_{i=1}^{n} \xi_{i}\left[I_{i}\right]_{\mathbb{U}},\left[I_{i}\right]_{\mathbb{U}} \in \boldsymbol{n b c}(\mathbb{U}), \xi_{i} \in \mathbb{K}$ and

(2) $[X]_{\mathbb{A}}=\sum_{i=1}^{n} \widetilde{\xi}_{i}\left[I_{i}\right]_{\mathbb{A}},\left[I_{i}\right]_{\mathbb{A}} \in \boldsymbol{n b c}(\mathcal{M}), \widetilde{\xi} \in\{ \pm 1,0\} \subset \mathbb{Z}$.

Then $\operatorname{sign}\left(\xi_{i}\right)=\widetilde{\xi}_{i}, i=1, \ldots, n$.

We make use of the following lemma:

Lemma 3.2 [5]. Let $G=(V, E)$ be the direct graph defined as follows:

$\circ \mathrm{V}(G)=\operatorname{IND}\left(\mathcal{M}\left(\mathcal{A}_{\mathbb{K}}\right)\right)$.

- $\overrightarrow{I I^{\prime}} \in \mathrm{E}(G)$ is a directed edge of $G$ iff there is a pivotable pair $(\alpha, x)$ such that $I^{\prime}=I \backslash x \cup \alpha$, where $\alpha=\alpha(I)$ and $x \in C(I, \alpha) \backslash \alpha$.

For every pair of vertices $X, X^{\prime}$ of the graph $G$, there is at most one directed path from $X$ to $X^{\prime}$.

Proof. We attach to $G$ two edge-labelling graphs determined respectively by the algebras $\mathbb{A}\left(\mathcal{M}\left(\mathcal{A}_{\mathbb{K}}\right)\right)$ and $\mathbb{U}\left(\mathcal{A}_{\mathbb{K}}\right)$. These labelled graphs are denoted $G_{\mathbb{A}}$ and $G_{\mathbb{U}}$, respectively. Let $\overrightarrow{I I_{i}^{\prime}}$ be an edge of $G$ where $\alpha=\alpha(I)=I_{i}^{\prime} \backslash I, C=C(I, \alpha)=$ $\left\{\alpha, x_{1}, \ldots, x_{i}, \ldots, x_{m}\right\}$ and $I_{i}^{\prime}=I \backslash x_{i} \cup \alpha$. Let $\boldsymbol{C} \in \mathfrak{C}\left(\mathcal{M}\left(\mathcal{A}_{\mathbb{K}}\right)\right)$ be the signed circuit supporting $C$ and such that $\boldsymbol{C}(\alpha)=1$.

(1) Suppose that $\partial(C)=e_{C \backslash \alpha}+\sum_{i=1}^{m} \zeta_{i} e_{C \backslash x_{i}}$, i.e., $[I]_{\mathbb{U}}=\sum_{i=1}^{m}-\zeta_{i}\left[I_{i}^{\prime}\right]_{\mathbb{U}}$. We label the edge $\overrightarrow{I I}_{i}^{\prime}$ of $G_{\mathbb{U}}$ with the scalar $-\zeta_{i}$.

(2) Suppose that $\widetilde{\partial}(C)=e_{C \backslash \alpha}+\sum_{i=1}^{m} \boldsymbol{C}\left(x_{i}\right) e_{C \backslash x_{i}}$ i.e., $[I]_{\mathbb{A}}=\sum_{i=1}^{m}-\boldsymbol{C}\left(x_{i}\right)\left[I_{i}^{\prime}\right]_{\mathbb{A}}$. We label the edge $\overrightarrow{I I}_{i}^{\prime}$ of $G_{\mathbb{A}}$ with the scalar $-\boldsymbol{C}\left(x_{i}\right)$.

From the definitions we know that $\operatorname{sign}\left(\zeta_{i}\right)=\boldsymbol{C}\left(x_{i}\right)$, for every $i=1, \ldots, m$. Let $\mathfrak{P}_{1}, \ldots, \mathfrak{P}_{s}$ be the list of the maximal length directed paths of $G$, beginning with the vertex $I$. Let $T_{\ell}$ denote the last vertex of the path $\mathfrak{P}_{\ell}, \forall \ell=1, \ldots, s . T_{\ell}$ is a sink of $G$, 
so $\left[T_{\ell}\right]_{\mathbb{A}} \in \boldsymbol{n b} \boldsymbol{c}(\mathcal{M})\left(\right.$ resp. $\left.\left[T_{\ell}\right]_{\mathbb{U}} \in \boldsymbol{n b} \boldsymbol{c}(\mathbb{U})\right)$. As $\mathbb{K}$ is an ordered field, the proposition follows.

Definition 3.3. Let $\mathbf{B}=\left\{b_{1}, \ldots, b_{n}\right\}$ be an arbitrary basis of $\mathbb{U}_{1}$ (resp. $\mathbb{A}_{1}$ ) such that the set $\operatorname{IND}\left(\mathcal{M}^{\prime}\right):=\left\{\left\{j_{1}, \ldots, j_{s}\right\} \subset[n]: b_{j_{1}} b_{j_{2}} \cdots b_{j_{s}} \neq 0\right\}$ is the family of the independent sets of a matroid $\mathcal{M}^{\prime}([n])$. We say that $\mathbb{U}$ fixes $\mathcal{M}\left(\mathcal{A}_{\mathbb{K}}\right)$ (resp. A fixes $\left.\underline{\mathcal{M}}\right)$ if the following condition holds:

○ There is a permutation $\sigma \in \mathfrak{S}_{n}$ and invertible scalars $\zeta_{i}$ such that $b_{i}=\zeta_{i} e_{\sigma(i)}, i=$ $1, \ldots, n$. (Note that $\sigma: \mathcal{M}^{\prime} \cong \mathcal{M}\left(\mathcal{A}_{\mathbb{K}}\right)\left(\right.$ resp. $\left.\sigma: \mathcal{M}^{\prime} \cong \underline{\mathcal{M}}\right)$.)

Proposition 3.4. In general we cannot reconstruct $\mathcal{M}$ from the "abstract algebra" $\mathbb{A}(\mathcal{M})$. In the case when the algebra $\mathbb{A}(\mathcal{M})$ fixes $\underline{\mathcal{M}}$ we can reconstruct the signed set of circuits $\mathfrak{C}(\mathcal{M})$, up to a reorientation and a permutation of the ground set $[n]$.

Proof. (The following example is similar to one of [6].) Consider the two direct graphs $\mathfrak{G}_{1}=\left(V_{1}, E_{2}\right)$ and $\mathfrak{G}_{2}=\left(V_{2}, E_{2}\right)$ (see Figs. 1 and 2):

○ $V_{1}:=\left\{v_{1}, \ldots, v_{5}\right\}$,

○ $E_{1}:=\left\{\boldsymbol{a}_{1}=\overrightarrow{v_{1} v_{2}}, \boldsymbol{a}_{2}=\overrightarrow{v_{2} v_{3}}, \boldsymbol{a}_{3}=\overrightarrow{v_{3} v_{1}}, \boldsymbol{a}_{4}=\overrightarrow{v_{4} v_{1}}, \boldsymbol{a}_{5}=\overrightarrow{v_{1} v_{5}}, \boldsymbol{a}_{6}=\overrightarrow{v_{5} v_{4}}\right\}$.

○ $V_{2}:=\left\{v_{1}, \ldots, v_{5}\right\}$,

$\circ E_{2}:=\left\{\boldsymbol{b}_{1}=\overrightarrow{v_{1} \vec{v}_{2}}, \boldsymbol{b}_{2}=\overrightarrow{v_{2} v_{3}}, \boldsymbol{b}_{3}=\overrightarrow{v_{4} \vec{v}_{3}}, \boldsymbol{b}_{4}=\overrightarrow{v_{3} v_{1}}, \boldsymbol{b}_{5}=\overrightarrow{v_{1} v_{5}}, \boldsymbol{b}_{6}=\overrightarrow{v_{5} v_{3}}\right\}$.

Let $\mathcal{M}_{\mathfrak{G}_{1}}$ (resp. $\mathcal{M}_{\mathfrak{G}_{2}}$ ) be the oriented matroid on the ground set $\{1, \ldots, 6\}$ determined by the graph $\mathfrak{G}_{1}\left(\right.$ resp. $\left.\mathfrak{G}_{2}\right)$. More precisely:

- $\mathcal{M}_{\mathfrak{G}_{1}}$ has two pairs of opposite signed circuits $\boldsymbol{C}_{1},-\boldsymbol{C}_{1}$ and $\boldsymbol{C}_{2},-\boldsymbol{C}_{2}$ where $\boldsymbol{C}_{1}^{+}=\{1,2,3\}, \boldsymbol{C}_{2}^{+}=\{4,5,6\}$ and $\boldsymbol{C}_{1}^{-}=\boldsymbol{C}_{2}^{-}=\emptyset$.

○ $\mathcal{M}_{\mathfrak{G}_{2}}$ has three pairs of opposite signed circuits $\boldsymbol{D}_{1},-\boldsymbol{D}_{1}, \boldsymbol{D}_{2},-\boldsymbol{D}_{2}$ and $\boldsymbol{D}_{3}$, $-\boldsymbol{D}_{3}$ where $\boldsymbol{D}_{1}^{+}=\{1,2,4\}, \boldsymbol{D}_{2}^{+}=\{4,5,6\}, \boldsymbol{D}_{1}^{-}=\boldsymbol{D}_{2}^{-}=\emptyset, \boldsymbol{D}_{3}^{+}=\{1,2$,$\} and$ $\boldsymbol{D}_{3}^{-}=\{5,6\}$.

Consider the algebras $\mathbb{A}=\mathbb{A}\left(\mathcal{M}_{\mathfrak{G}_{1}}\right)$ and $\mathbb{B}=\mathbb{A}\left(\mathcal{M}_{\mathfrak{G}_{2}}\right)$. From Definition 2.2 we know that $\mathbb{A}$ is the commutative $\mathbb{Z}$-algebra generated by the seven elements $1, e_{1}, \ldots, e_{6}$ and

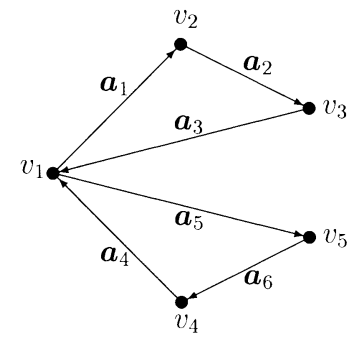

Fig. 1. Graph $\mathfrak{G}_{1}$.

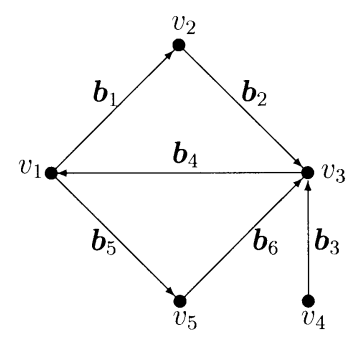

Fig. 2. Graph $\mathfrak{G}_{2}$. 
the relations

$$
e_{i} e_{j}=e_{j} e_{i}, \quad e_{12}+e_{13}+e_{23}=0, \quad e_{45}+e_{46}+e_{56}=0, \quad e_{i}^{2}=0
$$

for all $i=1, \ldots, 6$. Similarly, $\mathbb{B}$ is the commutative $\mathbb{Z}$-algebra generated by the seven elements $1, e_{1}^{\prime}, \ldots, e_{6}^{\prime}$ and the relations

$$
e_{i}^{\prime} e_{j}^{\prime}=e_{j}^{\prime} e_{i}^{\prime}, \quad e_{12}^{\prime}+e_{14}^{\prime}+e_{24}^{\prime}=0, \quad e_{45}^{\prime}+e_{46}^{\prime}+e_{56}^{\prime}=0, \quad e_{i}^{\prime} e_{i}^{\prime}=0
$$

for all $i=1, \ldots, 6$. (The relation $-e_{126}^{\prime}-e_{125}^{\prime}+e_{156}^{\prime}+e_{256}^{\prime}=0$ is redundant. Indeed, from the relations (3.2), we deduce that $-e_{126}^{\prime}=e_{146}^{\prime}+e_{246}^{\prime},-e_{125}^{\prime}=e_{145}^{\prime}+e_{245}^{\prime}$, $e_{156}^{\prime}=-e_{146}^{\prime}-e_{145}^{\prime}$ and $e_{256}^{\prime}=-e_{245}^{\prime}-e_{246}^{\prime}$.)

Let $\Phi: \mathbb{A} \rightarrow \mathbb{B}$ be the morphism of $\mathbb{Z}$-algebras determined by the values $\Phi(1)=1$, $\Phi\left(e_{\ell}\right)=e_{\ell}^{\prime}+e_{3}^{\prime}+e_{4}^{\prime}, \ell=1,2, \Phi\left(e_{3}\right)=-e_{3}^{\prime}$ and $\Phi\left(e_{\ell}\right)=e_{\ell}^{\prime}, \ell=4,5,6$. The map $\Phi$ is well defined. Indeed

$$
\begin{aligned}
\Phi\left(e_{12}+e_{13}+e_{23}\right) & =\left(e_{1}^{\prime}+e_{3}^{\prime}+e_{4}^{\prime}\right)\left(e_{2}^{\prime}+e_{3}^{\prime}+e_{4}^{\prime}\right)-e_{3}^{\prime}\left(e_{1}^{\prime}+e_{2}^{\prime}+2 e_{3}^{\prime}+2 e_{4}^{\prime}\right) \\
& =e_{12}^{\prime}+e_{24}^{\prime}+e_{14}^{\prime}=0,
\end{aligned}
$$

and

$$
\Phi\left(e_{45}+e_{46}+e_{56}\right)=e_{45}^{\prime}+e_{46}^{\prime}+e_{56}^{\prime}=0 .
$$

Consider now the morphism of $\mathbb{Z}$-algebras $\Theta: \mathbb{B} \rightarrow \mathbb{A}$ determined by the values $\Theta(1)=$ $1, \Theta\left(e_{\ell}^{\prime}\right)=e_{\ell}+e_{3}-e_{4}, \ell=1,2, \Theta\left(e_{3}^{\prime}\right)=-e_{3}$ and $\Theta\left(e_{\ell}^{\prime}\right)=e_{\ell}, \ell=4,5,6$. The map $\Theta$ is well defined. Indeed

$$
\begin{aligned}
\Theta\left(e_{12}^{\prime}+e_{14}^{\prime}+e_{24}^{\prime}\right) & =\left(e_{1}+e_{3}-e_{4}\right)\left(e_{2}+e_{3}-e_{4}\right)+e_{4}\left(e_{1}+e_{2}+e_{1}+2 e_{3}-2 e_{4}\right) \\
& =e_{12}+e_{13}+e_{23}=0,
\end{aligned}
$$

and

$$
\Theta\left(e_{45}^{\prime}+e_{46}^{\prime}+e_{56}^{\prime}\right)=e_{45}+e_{46}+e_{56}=0 .
$$

As $\Theta \circ \Phi=1_{\mathbb{A}}$ and $\Phi \circ \Theta=1_{\mathbb{B}}$, we conclude that the $\Phi$ is an isomorphism.

Suppose now that $\mathbb{A}=\mathbb{A}(\mathcal{M})$ fixes $\underline{\mathcal{M}}$ and let $e_{1}, \ldots, e_{n}$ be a family of generators of $\mathbb{A}_{1}$ in one-to-one correspondence $e_{i} \leftrightarrow i$ with the elements of the ground set $[n]$ of $\mathcal{M}$. Let $\boldsymbol{C}$ be the signed circuit of $\mathcal{M}$ supporting $\boldsymbol{C}=\left\{i_{1}, \ldots, i_{k}\right\}, k>1, i_{1}<\cdots<i_{k}$, and such that $\boldsymbol{C}\left(i_{1}\right)=1$. By a reordering of the elements of $[n]$ we may suppose that $i_{1}=1, \ldots, i_{j}=j$. Let $\boldsymbol{n b \boldsymbol { c }}(\mathcal{M})$ be the no broken circuit basis of the algebra $\mathbb{A}$ relative to this new ordering of $[n]$. It is clear that $[1 \cdots \widehat{j} \cdots k]_{\mathbb{A}} \in \boldsymbol{n b} \boldsymbol{c}(\mathcal{M})$, for every $j=2, \ldots, k$, but $\underline{\boldsymbol{C}} \backslash 1=\{2, \ldots, k\}$ is a broken circuit. We know that $[2 \cdots k]_{\mathbb{A}}=$ $-\sum_{j=2}^{k} \boldsymbol{C}(j)[1 \cdots \widehat{j} \cdots k]_{\mathbb{A}}$. So, we can recover the signed circuit $\boldsymbol{C}$. We conclude that the base $e_{1}, \ldots, e_{n}$ of $\mathbb{A}_{1}$ determines $\mathfrak{C}(\mathcal{M})$ up to a reorientation and a permutation of the ground set $[n]$.

Theorem 3.5. In general we cannot reconstruct the intersection lattice $L(\mathcal{A})$ from the "abstract algebra" $\mathbb{U}(\mathcal{A})$. 
Proof. Let $\boldsymbol{e}_{1}, \ldots, \boldsymbol{e}_{6}$ be the canonical basis of the vector space $\mathbb{R}^{6}$. Consider the graph $\mathfrak{G}_{1}=\left(V_{1}, E_{1}\right)$, introduced in Proposition 3.4. To every edge $\boldsymbol{a}_{h}=\overrightarrow{v_{i} \vec{v}_{j}} \in E_{1}$, we attach the vector $\boldsymbol{v}_{h}=\boldsymbol{e}_{i}-\boldsymbol{e}_{j}$. Set

$$
\mathcal{A}_{\mathbb{R}}=\left\{H_{\ell} \subset\left(\mathbb{R}^{6}\right)^{\star}: H_{\ell}=\left\{x \in\left(\mathbb{R}^{6}\right)^{\star}:\left\langle x, v_{\ell}\right\rangle=0\right\}, \ell=1, \ldots, 6\right\} .
$$

From the definitions, we see that $\mathcal{M}\left(\mathcal{A}_{\mathbb{R}}\right)=\mathcal{M}_{\mathfrak{G}_{1}}$. For every one of the four signed circuits $\boldsymbol{C} \in \mathfrak{C}\left(\mathcal{M}\left(\mathcal{A}_{\mathbb{R}}\right)\right)$ we have $\sum_{\ell=1}^{6} \boldsymbol{C}(\ell) v_{\ell}=0$. So, $\mathbb{U}\left(\mathcal{A}_{\mathbb{R}}\right)=\mathbb{A}\left(\mathcal{M}\left(\mathcal{A}_{\mathbb{R}}\right)\right) \otimes_{\mathbb{Z}} \mathbb{R}$. Similarly, we construct an arrangement of hyperplanes $\mathcal{B}_{\mathbb{R}}$ in $\left(\mathbb{R}^{6}\right)^{\star}$, determined by the graph $\mathfrak{G}_{2}=\left(V_{2}, E_{2}\right)$, and we also have $\mathbb{U}\left(\mathcal{B}_{\mathbb{R}}\right)=\mathbb{A}\left(\mathcal{M}\left(\mathcal{B}_{\mathbb{R}}\right)\right) \otimes_{\mathbb{Z}} \mathbb{R}$. So $\mathbb{U}\left(\mathcal{A}_{\mathbb{R}}\right) \cong \mathbb{U}\left(\mathcal{B}_{\mathbb{R}}\right)$ and $L\left(\mathcal{A}_{\mathbb{R}}\right) \varsubsetneqq L\left(\mathcal{B}_{\mathbb{R}}\right)$.

We finish with an open question. Orlik and Terao ask in [8]:

- Is $\mathbb{U}\left(\mathcal{A}_{\mathbb{C}}\right)$ the model for any topological invariant of the manifold $\mathfrak{M}\left(\mathcal{A}_{\mathbb{C}}\right)=$ $\mathbb{C}^{d} \backslash \bigcup_{H \in \mathcal{A}_{\mathrm{C}}} H$ ?

A partial solution to this problem can be obtained from Proposition 3.4 above and a celebrated theorem of Björner and Ziegler [3]:

Proposition 3.6. Suppose that $\mathcal{A}_{\mathbb{C}}$ is the complexification of a real arrangement $\mathcal{A}_{\mathbb{R}}$ and the algebra $\mathbb{U}\left(\mathcal{A}_{\mathbb{C}}\right)$ fixes the matroid $\mathcal{M}\left(\mathcal{A}_{\mathbb{C}}\right)$. Then the abstract algebra $\mathbb{U}\left(\mathcal{A}_{\mathbb{C}}\right)$ determines the smooth manifold $\mathfrak{M}\left(\mathcal{A}_{\mathbb{C}}\right)$ up to homeomorphism.

Proof. We know that $\mathbb{U}\left(\mathcal{A}_{\mathbb{C}}\right)=\mathbb{U}\left(\mathcal{A}_{\mathbb{R}}\right) \otimes_{\mathbb{R}} \mathbb{C}$ and $\mathcal{M}\left(\mathcal{A}_{\mathbb{C}}\right) \cong \mathcal{M}\left(\mathcal{A}_{\mathbb{R}}\right)$. From Proposition 3.4 we conclude that the algebra $\mathbb{U}\left(\mathcal{A}_{\mathbb{C}}\right)$ determines (up to reorientation and a permutation of the ground set $[n]$ ) the oriented matroid $\mathcal{M}\left(\mathcal{A}_{\mathbb{R}}\right)$. So the smooth manifold $\mathfrak{M}\left(\mathcal{A}_{\mathbb{C}}\right)$ is well determined up to homeomorphism, see [3].

A combinatorial analogue of the question of Orlik-Terao is:

$\circ$ Which features of the oriented matroid $\mathcal{M}$ are reflected in the algebra $\mathbb{A}(\mathcal{M})$ ?

\section{References}

1. Aomoto, K.: Hypergeometric functions, the past, today, and ... (from the complex analytic point of view) [translation of Sūgaku 45(3) (1993), 208-220]. Sugaku Expositions 9(1) (1996), 99-116.

2. Björner, A., Las Vergnas, M., Sturmfels, B., White, N., and Ziegler, G. M.: Oriented matroids, second edition. Encyclopedia of Mathematics and its Applications, vol. 46. Cambridge University Press, Cambridge, 1999.

3. Björner, A., and Ziegler, G. M.: Combinatorial stratification of complex arrangements. J. Amer. Math. Soc. 5(1) (1992), 105-149.

4. Brylawski, T.: The broken-circuit complex. Trans. Amer. Math. Soc. 234(2) (1977), 417-433.

5. Cordovil, R., and Etienne, G.: A note on the Orlik-Solomon algebra. European J. Combin. 22(2) (2001), 165-170.

6. Eschenbrenner, C. J., and Falk, M. J.: Orlik-Solomon algebras and Tutte polynomials. J. Algebraic Combin. 10(2) (1999), 189-199.

7. Orlik, P., and Terao, H.: Arrangements of Hyperplanes. Grundlehren der Mathematischen Wissenschaften, vol. 300. Springer-Verlag, Berlin, 1992. 
8. Orlik, P., and Terao, H.: Commutative algebras for arrangements. Nagoya Math. J. 134 (1994), 65-73.

9. White, N. (ed.): Theory of Matroids. Encyclopedia of Mathematics and its Applications, vol. 26. Cambridge University Press, Cambridge, 1986.

10. White, N. (ed.): Combinatorial Geometries. Encyclopedia of Mathematics and its Applications, vol. 29. Cambridge University Press, Cambridge, 1987.

Received November 7, 2000, and in revised form May 18, 2001. Online publication November 2, 2001. 